\title{
La conservación del bosque en Tetela del Volcan, Morelos, vista a través de un análisis situacional
}

\author{
Juan Manuel Chávez-Cortés \\ Gabriela Ramírez Granados ${ }^{1}$ \\ Marisol Medina ${ }^{1}$
}

RESUMEN

\begin{abstract}
La necesidad de involucrar en la toma de decisiones a todos los actores sociales en el manejo de ecosistemas forestales, es considerada como uno de los pilares fundamentales en el manejo forestal sustentable (MFS). Sin embargo, también se le identifica como el pilar "más débil", principalmente cuando se requiere de la colaboración de múltiples actores sociales. El buscar esta colaboración significa entender y conocer las percepciones y la manera en que pueden influir los usuarios y los dueños acerca del manejo de los bosques, así como la identificación de los problemas o conflictos inmersos en el mismo. En este trabajo se postula que plantear propuestas de cómo contender con problemas y tendencias contrarias al MFS, obliga a hacer un análisis situacional de la problemática y la disponibilidad a colaborar de los actores sociales. Para probar este postulado se emplea una estrategia de investigación de estudio de caso: el municipio de Tetela del Volcán, Morelos, con el objetivo de identificar la problemática y las potencialidades para desarrollar el diseño de un programa de conservación forestal para este municipio. Los autores identificaron como la principal problemática local para el logro del MFS a "Ia falta de cultura acerca de la valoración de los recursos forestales en la población". Para contender con esta problemática se construyeron dos programas de acciones prioritarias, encaminados a mejorar el presupuesto del municipio destinado a la conservación y reforestación y promover la coordinación, comunicación y concertación entre las diferentas autoridades para el manejo de los recursos forestales.
\end{abstract}

PALABRAS CLAVE:

Análisis situacional, manejo forestal sustentable, participación de actores sociales múltiples, planeación estratégica, programa de acciones prioritarias.

\begin{abstract}
Multi-stakeholders involvement in forest ecosystems management is considered as one of the main pillars in Sustainable Forest Management (SFM). However, it is also seen as the "weak pillar" because of the required collaboration of multiple-stakeholders. In order to obtain this collaboration, the planner has to understand the perceptions and the ways different stakeholders influence forest management. He also has to identify the problems and conflicts related to this management. This paper states that a searching for alternatives to face problems related to the achievement of SFM requires situational (context) analysis of the stakeholders involved in forest management. In addition, a case study as research strategy is employed for testing this postulate. For this reason, the factors that foster and restrict a forest conservation project for SFM planning purposes in the municipality of Tetela del Volcán, Morelos, México, are analyzed and employed. The authors identified "the lack of a valuing culture of forest resources" as the main problem to achieve MFS. In order to tackle this complex problem, two strategic actions programs are built. One related to improve the budget for forest conservation and restoration, and other to empower the communication, coordination and negotiation of the stakeholders of the municipality.
\end{abstract}

KEYWORDS:

Multi-stakeholders involvement, situational analysis, strategic actions program, strategic planning, sustainable forest management.

Universidad Autónoma Metropolitana-Xochimilco. Departamento El Hombre y su Ambiente. Calzada del Hueso 1100, Villa Quietud, 09410 Coyoacan, México, D.F. ce: ccjm1308@correo.xoc.uam.mx 


\section{INTRODUCCIÓN}

En el contexto del discurso del desarrollo sustentable, el manejo forestal sustentable (MFS) se caracteriza fundamentalmente por la integración de la conservación del bosque y el desarrollo económico y social. Esta integración se busca que dé lugar a procesos que activen la búsqueda del logro de objetivos múltiples relacionados con la sustentabilidad ecológica, económica y social, en donde además, se le dé énfasis a los objetivos relacionados con la producción de un flujo continuo de productos y servicios ambientales derivados de los ecosistemas forestales (Hartshorn, 1995; Higman et al., 1999).

Por otro lado, desde una perspectiva más ligada a la concepción de sustentabilidad, promovida por la comunidad científica forestal, se reconoce que el mayor logro de esta estrategia de manejo se asocia a la integración del conocimiento tanto de los científicos y técnicos como de los usuarios de los bosques, lo cual significa incluir a todos los grupos de interés en el manejo de los bosques. Otro punto a resaltar de este enfoque es la consideración del manejo de los ecosistemas forestales a escalas temporales y espaciales grandes (manejo de ecosistemas) (Hartshorn, 1995; Yafee et al., 1996; Naiman et al, 1997).

Dentro de los criterios que han sido utilizados para definir MFS destacan aquellos que tienen que ver con el mejoramiento y mantenimiento de la biodiversidad, la concepción multifuncional de los ecosistemas forestales, la rentabilidad económica y la necesidad de considerar a todos los actores sociales involucrados en el manejo de ecosistemas forestales en la toma de decisiones (Mendoza y Prabhu, 2000).

La conservación de la biodiversidad se ve como una de los pilares del MFS debido a que se reconoce la importancia que tiene en el mantenimiento a largo plazo de los flujos de bienes y servicios que proporcionan los ecosistemas forestales. Asimismo, la biodiversidad es reconocida como la base de la estructura de estos ecosistemas pues permite que los mismos respondan adaptativamente a las perturbaciones naturales o causadas por el hombre. De hecho, se le atribuye dar lugar a las capacidades de resiliencia y homeorresis que estos ecosistemas desarrollan (Christensen et al., 1996). De allí que, el MFS deba considerar a la conservación de la biodiversidad como un prerrequisito.

Otro pilar fundamental del MFS es la concepción multi-funcional de los ecosistemas forestales (Lämas y Ola, 2003). Esta visión contrasta significativamente con la tradicional, la cual ve a los ecosistemas forestales sólo como fuente de productos maderables o de pulpa para celulosa. Por el contrario, la visión multi-funcional además de la visión anterior considera a los bosques como fuente de otros bienes y servicios. Entre ellos destacan la de productos no maderables (hongos, plantas de ornato, animales silvestres, plantas medicinales, entre otros). Para el caso de los servicios, destacan la producción de agua pura, la formación de suelo, la limpieza del aire y la disponibilidad de escenarios con una belleza particular que permiten la recreación en contacto con la naturaleza. Desde una perspectiva ética, algunos manejadores de bosques los ven como "los guardianes del patrimonio agua y suelo de una nación" (Kohn y Franklin, 1997; Higman et al., 1999; Lämas y Ola, 2003).

Ligado al punto anterior está un tercer pilar del MFS, el de la rentabilidad económica. La visión multifuncional está estrechamente ligada a la de diversidad de oferta e ingresos por bienes y servicios de los ecosistemas forestales. Si bien los productos maderables siguen siendo el principal producto de los ecosistemas forestales con un mercado establecido, en las dos últimas décadas se empiezan a desarrollar otros mercados que le confieren una mayor rentabilidad a los bosques. Dentro de los mismos cabe destacar el de turismo en contacto con la naturaleza, en donde los bosques ofertan "naturaleza" para un sinnúmero de actividades de tipo recreativo y educativo (Constanza et al., 1997; de Groot et al., 2002). Otro mercado que está surgiendo y cobra particular importancia es la de productos no 
maderables, destacando el de la venta de organismos como hongos, mariposas, aves, pequeños mamíferos, orquídeas, entre otros. Un tercer mercado, que actualmente está desarrollándose en forma vigorosa en el último lustro, es el de la venta de servicios ambientales (de Groot et al., 2002). Asimismo en muchas regiones del mundo la escasez de agua pura ha desarrollado el interés de la sociedad por la conservación de los bosques como fuente y reguladores del ciclo hidrológico, dando lugar a una creciente disponibilidad a pagar por mantener los bosques como una garantía del abasto de agua dulce regional (Naiman et al., 1997), aunque cabe aclarar aquí, que una premisa del MFS es que la conservación de los bosques debe estar apoyada en su rentabilidad, es decir, los bosques no pueden ser defendidos sólo a través de la internalización de externalidades.

El cuarto pilar del MFS es el de la necesidad de considerar a todos los actores sociales involucrados en el manejo de ecosistemas forestales en la toma de decisiones. EI MFS considera que la viabilidad social de los proyectos de manejo de ecosistemas forestales descansa en la participación de los actores sociales. Lo anterior lleva al obligado reconocimiento de que el MFS requiere, a su vez, de organizaciones sustentables involucradas en su conservación y aprovechamiento. Es decir, contar con organizaciones comprometidas, bien informadas, bien estructuradas, con habilidades de gestión y con profundas raíces en la sociedad local. Asimismo, capaces de establecer relaciones intersectoriales y con diferentes instancias de gobierno. Autores como Yaffee et al.,1996) consideran que este pilar es el más importante para garantizar el éxito en proyectos de MFS. De hecho, estos autores recomiendan el involucramiento de todos los actores sociales desde el inicio de cualquier proyecto de MFS.

Sin embargo a pesar de su importancia, la dimensión social también ha sido reconocida como el pilar "más débil" del MFS, principalmente cuando se requiere de actores sociales múltiples dispuestos a colaborar. Por ello, la literatura académica ha puesto atención al papel de las instituciones, la gobernancia y la disposición social a colaborar en el proceso de lograr el MFS. Es importante resaltar que la identificación de los problemas o conflictos inmersos en el MFS no es suficiente, que es necesario entender la percepción que tienen los usuarios y dueños acerca del manejo de los bosques. Muchos malos entendidos y mitos que predisponen a los usuarios y a dueños a actuar de determinadas maneras surgen de este análisis. Por esta razón, es importante entender y conocer las percepciones y la manera en que pueden influir en el MFS, ya que son el factor restrictivo que limita muchas veces las potencialidades de construir propuestas de participación que involucren actores múltiples en el MFS (adaptado de Ruelas, 2004). Desde la perspectiva de la planeación, distintos enfoques han sido empleados para contender con los problemas o conflictos inmersos en el MFS; destacan el enfoque colaborativo (Selin y Chavez, 1995; Healey, 1997), la planeación estratégica situacional (Matus, 1989) y el análisis de fortalezas, oportunidades, debilidades y amenazas (FODA) (Kajanus, Kangas y Kurttila, 2004; Hill y Westbrook, 1997; Pesonen, Kurttila, Kangas, Kajanus y Heinonen, 2001). Desde una perspectiva científico-técnica en la construcción del discurso de la sustentabilidad, el análisis situacional de Matus (1989) y el análisis FODA permiten una primera visión, sistemática, integral y amplia de la problemática inmersa en el proceso de lograr el MFS involucrando instituciones, la disposición social a colaborar y la identificación de los momentos de oportunidad y los factores restrictivos. Además, estos enfoques propios de la planeación estratégica, permiten generar directrices para la construcción de las propuestas de solución.

En México, el MFS es visto como una estrategia deseable y como la base para establecer las normas para alcanzarlo. El país cuenta con una Ley para el Manejo Sustentable de los Bosques, pero con escasas probabilidades de aplicación, dado el pobre o nulo apoyo en recursos financieros y humanos para promoverlo. Por otro lado, las 
condiciones de organización y cultura forestal locales (municipio, comunidad o ejido forestal) hacen de difícil realización las propuestas hacia un MFS. Los autores de este trabajo postulan que la cultura y las estrategias para el futuro que tienen los tomadores de decisiones involucrados en el manejo forestal, en el ámbito local, es aún uno de los mayores retos para la promoción del MFS. Esta investigación hace suyo este reto y para ello, se emplea una estrategia de investigación de estudio de caso. Por esta razón, se seleccionó al municipio de Tetela del Volcán, Morelos, por su importancia regional en el área de influencia del Parque Nacional Iztaccihuatl.-Popocatépetl. Este municipio contiene más del $50 \%$ de cubierta vegetal de la parte alta de la subcuenca de Huautla, y en la última década esta cubierta ha sido afectada por una serie de factores, entre los que destacan su tendencia al cambio de uso de suelo agrícola (fruticultura de durazno), alta incidencia de incendios, tala clandestina y proliferación de asentamientos irregulares (Chávez, 2004). Plantear propuestas de cómo contender con estos problemas y tendencias, contrarias a cualquier propuesta hacia la sustentabilidad, obliga a hacer un análisis situacional de la problemática local y la disponibilidad de los actores sociales del municipio a colaborar en la conservación de los bosques.

\section{OBJETIVOS}

El objetivo general de este trabajo fue identificar la problemática y las potencialidades para desarrollar el diseño de un programa de conservación forestal para el municipio de Tetela del Volcán.

Con base en el razonamiento anterior, se establecieron como objetivos particulares:

a) Identificar a los actores sociales del municipio involucrados en el manejo forestal y sus percepciones.

b) Identificar las potencialidades $y$ amenazas del contexto regional para llevar acabo la construcción del citado programa.

\section{METODOLOGÍA}

Para el desarrollo de este trabajo se seleccionó el esquema metodológico de planeación regional sustentable propuesto por Botequilha y Ahern (2002). La racionalidad detrás de su selección tiene que ver con criterios de integralidad, direccionalidad y flexibilidad. En opinión de los autores, es hasta la fecha el esquema que mejor integra las fases de la planeación regional, como son enfoque, análisis, diagnóstico, prognosis y sindéresis y a la vez, da una visión completa del proceso de planeación. Asimismo, da énfasis en la fase de enfoque al proceso de planteamiento de los problemas y de las metas que le darán direccionalidad al resto de las fases. Finalmente, introduce la fase de sindéresis, la cual tiene que ver con el diseño del mecanismo de seguimiento y evaluación del plan que garanticen una influencia de los resultados del mismo sobre sus objetivos iniciales. Esto último asegura la cualidad de flexibilidad en los planes. En este trabajo sólo se aplicaron las cuatro primeras, las cuales se describen a continuación.

\section{Fase de enfoque}

Esta fase consiste en la identificación de metas, propósitos y objetivos del tipo de trabajo que se va a realizar. Tal es el caso en esta investigación, donde se busca evaluar las potencialidades para un manejo forestal hacia la sustentabilidad en un ámbito local.

\section{Fase de análisis}

Esta fase se diseñó para ser abordada en cinco etapas: Identificación de actores sociales, elaboración del guión para la entrevista, aplicación de la entrevista, análisis de la entrevista y por último, la clasificación y agrupación de problemas. Cada una de las etapas se describe a continuación.

\section{- Identificación de actores sociales}

En esta etapa se identificaron a los actores involucrados en el manejo de los recursos forestales del municipio que iban a ser entrevistados. La selección se dió con 
base en una charla con el regidor de Ecología y Hacienda, quien guió la selección de los actores locales y externos que tradicionalmente han influido en la toma de decisiones sobre la conservación y restauración del bosque.

\section{entrevista}

- Elaboración del guión para la

Aquí se elaboró el guión de la entrevista, basada en un cuestionario que contenía aquellas preguntas relacionadas principalmente al manejo de los recursos forestales, con el fin de identificar si existen programas de reforestación, cómo se aplican, sus alcances, hasta donde se aplican, así como la identificación de actores sociales que puedan ser aliados para la promoción del MFS. El cuestionario se describe en la Nota 1.

\section{- Aplicación de la entrevista}

Una vez elaborado el guión para aplicar en las entrevistas, se requirió de, al menos dos personas para la realización de las entrevistas a cada actor social, de tal manera que una dirigiera la entrevista siguiendo la secuencia de preguntas, en tanto la otra se dedicase a tomar nota de la información proporcionada por los actores.

\section{- Análisis y síntesis de la entrevista}

En esta fase se identificaron los problemas derivados de las entrevistas y se realizó un listado de los mismos. Asimismo se escogió como problemática forestal local a la falta de un programa de conservación forestal en el Municipio de Tetela del Volcán.

\section{problemas}

- Clasificación y agrupación de

En esta fase se elaboró un cuadro de problemas, con base en dos categorías propuestas por Matus (1989): una explicada por el grado de afectación, el cual se refiere a identificar el grado con que se afectan grupos sociales, es decir, si afecta a un sólo individuo de un grupo, entonces afecta de manera particular; a un grupo, afecta de manera singular o a todos los grupos, es decir, de manera general; y otra relacionada con la tipología del problema, ligada a la naturaleza de los problemas, fenomenológicos, estructurales o genoestructurales (Matus, 1989).

\section{Fase de diagnóstico}

En esta fase, los problemas seleccionados son arreglados buscando establecer la sinergia entre ellos. La finalidad es la de identificar en esta trama de problemas aquellos que muestran un mayor número de relaciones, los cuales se identifican como los nodos problemáticos. Posteriormente son separados de la trama y se acomodan en una estructura denominada árbol problemático (Matus, 1989).

\section{Fase de prognosis}

Esta fase se diseñó considerando las siguientes etapas: - Establecimiento de ejes
estratégicos

A cada nodo problemático se le asocia una propuesta de dirección estratégica que permita contender con las problemáticas identificadas. Estas directrices estratégicas son denominadas ejes estratégicos.

\section{- Establecimiento de objetivos estratégicos}

En esta etapa se definen las tácticas para lograr los propósitos establecidos en cada uno de los ejes estratégicos.

\section{estratégicas \\ - Establecimiento de acciones}

Esta etapa se caracteriza por ser de naturaleza táctico-operativo. En la misma se privilegian las acciones que complementan a las reflexiones derivadas de la fase de diagnóstico. En ella se establecen cuatro grandes rubros: tipos de acciones a realizar, la identificación de los actores encargados de coordinar el logro de las acciones, identificar a los actores sociales responsables de realizar las actividades, y la estructuración de un 
calendario de actividades.

\section{- Análisis de contexto}

En esta etapa se aplica una evaluación de las amenazas y potencialidades para el logro del MFS a que el contexto local da lugar. Para esta evaluación se aplica parcialmente, el enfoque metodológico del análisis FODA.

\section{RESULTADOS}

\section{Fase de enfoque}

En esta fase, se establecieron el objetivo general y los objetivos particulares que le dieron dirección a la presente investigación. Ambos están descritos en detalle en la última parte de la introducción.

\section{Fase de análisis}

\section{Identificación de actores sociales}

En esta fase se identificó a los actores sociales involucrados en el manejo de los recursos forestales del municipio y los recursos (financieros, de organización, de convocatoria, etc.) bajo su control. Estos actores y los recursos que controlan están enumerados en la Tabla 1. Los actores sociales responsables de la elaboración de un plan de manejo forestal en el municipio, el regidor de Ecología y Hacienda, los Comisariados de Bienes Comunales de la cuatro comunidades del municipio, los cuatro Comisariados Ejidales y el regidor de Protección Civil fueron entrevistados, siguiendo la guía para la entrevista previamente diseñado (Nota 1).

\section{Análisis de las entrevistas}

Los datos obtenidos a partir de las entrevistas aplicadas a los actores sociales descritos arriba, se analizaron a través de un procedimiento de agrupación y jerarquización. Primeramente, se agruparon las opiniones por similitud y se seleccionó a la definición del problema y a la frase testimonio que mejor las ilustraba. Posteriormente se hizo un análisis de frecuencia, el cual permitió establecer una jerarquía de las opiniones. A

Nota 1. Preguntas guía para las entrevistas a los involucrados en el manejo forestal municipal

1. ¿El municipi o cuenta con al gún programa de conservación forestal?

2. ¿Se aplica el programa?

3. ¿Existe el apoyo económico por parte de alguna Institución?

4. ¿El presupu esto destinado al programa, es suficiente?

5. ¿El personal encargado para la aplicación del programa, es suficiente?

6. ¿El personal se encuentra capacitado?

7. Si se han hech o programas de conservación forestal anteriormente, ¿Han sido aceptados?

8. ¿Qué deficienci as encuentran en el programa?

9. ¿Qué recomi enda para que la a plicación del programa s ea efectiva?

10. ¿Existe algún moni toreo para que se aplique adecuadamente el programa?

11. ¿Cada cuánto se hace el monitor eo?

12. ¿Qué modificaciones sugiere para que el programa se mejore?

Previendo el caso de que el municipio no contase con un programa de conservación forestal, se elaboró un guión que contiene las siguientes preg untas:

1. ¿Estarían de acuerdo en la introducción y aplicación de un programa de conservación forestal?

2. ¿Dónde estarían de acuerdo en que se aplicará el programa?

3. ¿A quién les pedirían apoyo económico?

4. ¿Cómo les gustaría que fuera el programa?

5. ¿Qué están de acuerdo en con servar?

6. ¿Cuáles serían los problemas más importantes para aplicar el plan de conservación forestal? 
Tabla 1. Actores sociales involucrados y el recurso que manejan

ACTORES SOCIALES

RECURSOS BAJO SU CONTROL

Regidor de Ecología y Hacienda del municipio Gestión y obtención de recursos económicos y forestales. de Tetela del Vol cán, Mor.

\begin{tabular}{ll}
\hline Regidor de Protección Civil & Vigilancia y prevención contra incendios forestales. \\
\hline Comisario Ejidal & Gestión entre los ejidatarios para el uso agrario. \\
\hline Comis ario de Bienes Com unales & $\begin{array}{l}\text { Comunicación-concertación y coordinación entre las } \\
\text { comuni dades del Munici pio. }\end{array}$ \\
\hline Planeador de la UAM-X & Capacidad de elaborar planes. \\
\hline Destacamento local del Ejército Mexicano & Mano de obra y transporte \\
\hline $\begin{array}{l}\text { Instituciones federales y estatal es } \\
\text { relacionadas con el medio ambiente y manejo } \\
\text { forestal (CONAFOR, SEDESOL) }\end{array}$ & Apoyo financiero \\
\hline $\begin{array}{l}\text { Instituciones no gubernamentales (ONGs de } \\
\text { carácter nacional e internacional) }\end{array}$ & Apoyo financiero \\
\hline $\begin{array}{l}\text { Instituciones educativas ( primarias y } \\
\text { secundarias locales) }\end{array}$ & Apoyos de mano de obra para la reforestación \\
\hline
\end{tabular}

Tabla 2. Problemática y listado de problemas, detectados en el Municipio de Tetela del Volcán

Problemática
Falta de una cultura que valorice apropia damente los recursos for estales en el Muni cipio de Tetel a del Volcán
Problemas


partir de este análisis se elaboró un listado de problemas que definen la problemática sobre la conservación forestal en el municipio. A la problemática los autores la definieron como la falta de una cultura que valorizara apropiadamente los recursos forestales del municipio (ver Tabla 2).

Clasificación y agrupación de problemas detectados

Una vez identificada la problemática que explica los problemas en el municipio, se elaboró un cuadro de problemas, con base a la clasificación propuestas por Matus (1989) basado en el grado de afectación y la tipología de los problemas (ver tabla 3 ).

\section{Fase de diagnóstico}

Con base en la clasificación y agrupación de los problemas de la Tabla 3, se estructuró el árbol problemático, el cual describe la sinergia de causalidad entre ellos. Con base en el número de relaciones causales se identificaron dos nodos problemáticos que, en opinión de los autores, explican la problemática (Figura 1).

De la aplicación parcial del análisis

Tabla 3. Clasificación y agrupación de problemas

\begin{tabular}{|c|c|c|c|}
\hline \multirow{2}{*}{$\begin{array}{l}\text { TIPOLOGÍA DEL } \\
\text { PROBLEM A }\end{array}$} & \multicolumn{3}{|c|}{ GRADO DE AFECTACIÓN } \\
\hline & GENERALES & PARTICULARES & SINGULARES \\
\hline FENOMENOLÓGICO & & $\begin{array}{l}\text { - No hay vigilancia y } \\
\text { mantenimi ento en las zonas } \\
\text { reforestadas por parte del } \\
\text { municipio y PROFEPA. } \\
\text { - No hay recon ocimiento de los } \\
\text { límites del municipio de parte } \\
\text { de Ocuituco y Hueyapan lo } \\
\text { que da lugar a que haya un } \\
\text { aprovechamiento libre del } \\
\text { recurso. } \\
\text { No hay una aplicación de la } \\
\text { Ley Forestal por parte de los } \\
\text { comisarios de Bienes Comu- } \\
\text { nales por mi edo a represalias. } \\
\text { Poca participación ciudadana, } \\
\text { para reforestar y contender } \\
\text { contra incendios, además de } \\
\text { la escasa asistencia a cursos } \\
\text { destinados a las actividades } \\
\text { anteriores }\end{array}$ & \\
\hline ESTRUCTURAL & $\begin{array}{l}\text { - Falta de com unicación y } \\
\text { unificación de ideologías } \\
\text { entre los diferentes } \\
\text { niveles de gobierno para } \\
\text { el manejo de los recursos } \\
\text { forestal es. } \\
\text { - Falta de Presupuesto por } \\
\text { parte del Muni cipio } \\
\text { destinado a la refores- } \\
\text { tación y cons ervación. }\end{array}$ & & \\
\hline GENO-ESTRUCTURAL & $\begin{array}{l}\text { - No hay un Programa de } \\
\text { Conservación Forestal } \\
\text { por parte del Muniapio }\end{array}$ & & \\
\hline
\end{tabular}


FODA, con el propósito de mejorar la visión del contexto local, se identificaron las siguientes amenazas y oportunidades:

\section{Amenazas}

1. La poca participación que existe por parte de las comunidades del municipio para la asistencia a cursos enfocados a la prevención de incendios y la participación para la reforestación. A pesar de que Protección Civil promueve y realiza cursos enfocados a la prevención de incendios, los habitantes del municipio se niegan a participar si no hay una compensación monetaria. Asimismo consideran una pérdida

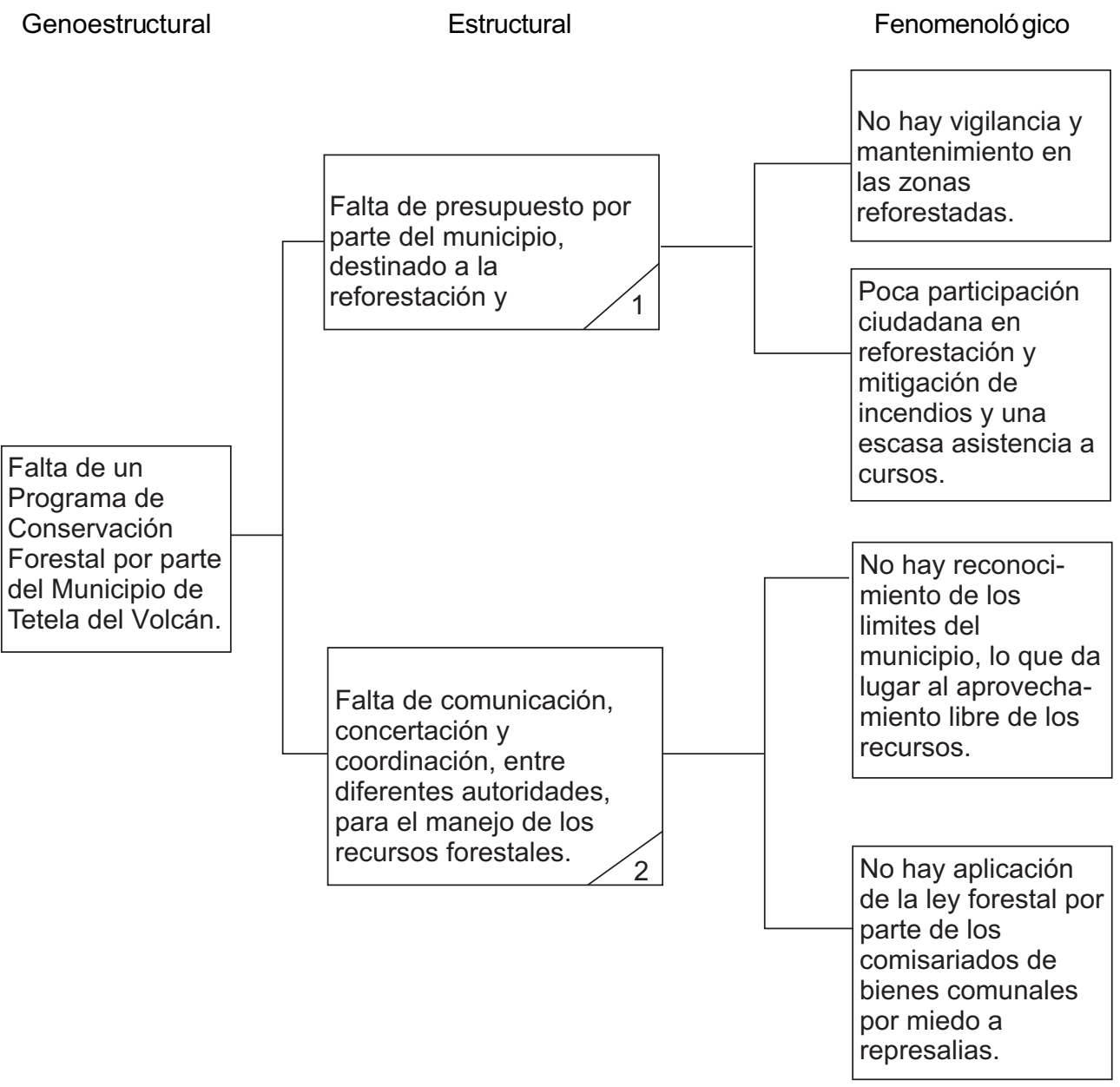

Figura 1. Árbol problemático 
de tiempo asistir a cursos y al apoyo de acciones de reforestación si no hay una remuneración.

2. El Regidor de Ecología y Hacienda, respecto al desarrollo ecoturístico, busca beneficios únicamente para su comunidad y no para toda la población de Tetela del Volcán, por lo que descuida las potencialidades que se dan en otras comunidades del municipio dejando de lado su promoción y desarrollo.

\section{Oportunidades}

1. Hay disponibilidad para la participación en el Programa de Conservación Forestal por parte del Comisariado de Bienes Comunales. Existe una gran preocupación del mismo por la carencia de una política ambiental eficiente en el municipio, en donde las mayores perturbaciones corresponden a los recursos forestales que se han visto disminuidos por las fuertes presiones en los bosques como ya se mencionó en la introducción.

2. Hay aceptación por parte del Regidor de Ecología y Hacienda para la creación de zonas ecoturísticas, que fomente el desarrollo del municipio. Esta propuesta se considera generadora de fuentes de trabajo para los habitantes del mismo. Tal es el caso de la comunidad de Tlalmimilulpan, en la cual se destinó una zona para la construcción de una cabaña-restaurante, la que ha generado fuentes de trabajo y promovido a pequeña escala el ecoturismo.

\section{Fase de prognosis}

\section{Establecimiento de Ejes Estratégicos}

A partir de los nodos problemáticos se generó una serie de propuestas que se consideran de carácter estratégico para construir las propuestas de solución con un carácter normativo (deber ser). Para ello se estableció un propósito que guiara las propuestas para la solución al problema identificado en cada nodo. A estos propósitos se les denominaron ejes estratégicos.

Para el nodo 1. Falta de presupuesto por parte del municipio, destinado a reforestación y conservación, se propuso como eje estratégico:

Mejorar y aumentar el presupuesto del Municipio destinado a la conservación y la reforestación.

Para el nodo 2. Falta de comunicación, coordinación y concertación entre diferentes autoridades para el manejo de recursos forestales, se propuso como eje estratégico el:

Promover y mejorar la coordinación, comunicación y la concertación entre las diferentes autoridades, para el manejo de los recursos forestales.

Posteriormente, con la finalidad de darle un carácter operativo y táctico a cada uno de los ejes se elaboraron programas de acciones prioritarias. En los mismos, además de la descripción de lo ejes estratégicos se incluyeron objetivos de trabajo, acciones estratégicas, los coordinadores y colaboradores en la realización de estas actividades (tabla 4).

\section{DISCUSIÓN DE LOS RESULTADOS}

En términos generales, se puede afirmar que la selección de la estrategia de investigación y de la metodología fue apropiada para contender con las necesidades de análisis del cuarto pilar del MFS: considerar a los actores sociales involucrados en el manejo de ecosistemas forestales en la toma de decisiones. Por un lado, el estudio de caso, como estrategia de investigación, se perfila como la apropiada para abordar la reflexión-acción requeridas en problemáticas en donde el contexto es clave en el diagnóstico y en las propuestas. A su vez, los resultados dejan ver las bondades del esquema metodológico para la planeación regional sustentable propuesto por Botequilha y Ahern (2002). En particular, sobresalen las posibilidades que brinda para una integración sistemática de diferentes métodos y procedimientos y la de dar lugar a una visión completa y sinérgica del estudio. 
Una mención particular, se debe de dar a la integración que en este trabajo se hizo del análisis situacional (Matus, 1989) que de lugar a vías hacia el MFS. En opinión de los autores se perfila como una de los procedimientos más oportunos y sólidos en el análisis de los actores sociales y su contexto,

Tabla 4. Plan de Acciones Prioritarias

\begin{tabular}{|c|c|c|c|}
\hline $\begin{array}{l}\text { OBJETIVOS } \\
\text { ESTRATÉGICOS }\end{array}$ & ACCIONES ASOCIADAS & $\begin{array}{l}\text { QUIÉN } \\
\text { COORDINA }\end{array}$ & QUIÉN REALIZA \\
\hline \multirow[t]{2}{*}{$\begin{array}{l}\text { Diversificar las fu entes } \\
\text { de financiam iento para } \\
\text { la promoción de zonas } \\
\text { forestal es del municipio } \\
\text { destinadas al } \\
\text { ecoturismo. }\end{array}$} & $\begin{array}{l}\text { Convocar a las dep endencias } \\
\text { gubernamentales y no } \\
\text { gubernamentales, para } \\
\text { construir aquerdos de } \\
\text { promoción a las zonas } \\
\text { dirigidas al ecoturismo. }\end{array}$ & \multirow[t]{2}{*}{$\begin{array}{l}\text { Regidor de } \\
\text { Ecología y } \\
\text { Hacienda }\end{array}$} & $\begin{array}{l}\text { Regidor de Ecología y } \\
\text { Hacienda, CONAFOR, } \\
\text { SEDESOL, Ramo 33, } \\
\text { SAGARPA e Instituciones no } \\
\text { gubernamentales. }\end{array}$ \\
\hline & $\begin{array}{l}\text { Buscar presup uesto para la } \\
\text { creación de empl eos } \\
\text { destinados a la vigilancia y al } \\
\text { monitoreo de las áreas } \\
\text { for estal es. }\end{array}$ & & Regidor de Ecología y Hacienda \\
\hline \multirow{5}{*}{$\begin{array}{l}\text { Involucrar a las } \\
\text { escuelas local es en el } \\
\text { proceso de } \\
\text { reforesta ción }\end{array}$} & $\begin{array}{l}\text { Convocar a los dir ectores de } \\
\text { las escuelas }\end{array}$ & \multirow{5}{*}{$\begin{array}{l}\text { Regidor de } \\
\text { Ecología y } \\
\text { Hacienda }\end{array}$} & Regidor de Ecologí a y Hacienda \\
\hline & $\begin{array}{l}\text { Implementar cursos de } \\
\text { educación ambi ental }\end{array}$ & & Planeador y Prof esores \\
\hline & $\begin{array}{l}\text { Construir acu erdos para la } \\
\text { reforesta ción }\end{array}$ & & $\begin{array}{l}\text { Regidor de Ecología y } \\
\text { Hacienda, Di rectores de las } \\
\text { instituciones educativas y } \\
\text { profesores }\end{array}$ \\
\hline & $\begin{array}{l}\text { Conseguir vehí culos para } \\
\text { transportar a los alumnos }\end{array}$ & & Ejército y las comunidades \\
\hline & $\begin{array}{l}\text { Conseguir herr amientas para } \\
\text { la siembra de plántulas }\end{array}$ & & $\begin{array}{l}\text { Viveros, Ejército, Prot ección } \\
\text { Civil y Comunidades }\end{array}$ \\
\hline \multirow[t]{5}{*}{$\begin{array}{l}\text { Involucrar a Prot ección } \\
\text { Civil y al Ejército para } \\
\text { la prevención de } \\
\text { incendios }\end{array}$} & $\begin{array}{l}\text { Convocar a prot ección civil, al } \\
\text { ejército y a las comunidades. }\end{array}$ & \multirow[t]{5}{*}{$\begin{array}{l}\text { Regidor de } \\
\text { Ecología y } \\
\text { Hacienda y } \\
\text { Protección Civil }\end{array}$} & $\begin{array}{l}\text { Protección Civil, Ejército, } \\
\text { Comisariado de Bienes } \\
\text { Comunales, Com isariado Ejidal } \\
\text { y las Comunid ades }\end{array}$ \\
\hline & $\begin{array}{l}\text { Construir acu erdos para el } \\
\text { control y pr evención de } \\
\text { incendios. }\end{array}$ & & $\begin{array}{l}\text { Protección Civil, Ejército, } \\
\text { Comisariado de Bienes } \\
\text { Comunales, Com isariado Ejidal } \\
\text { y las Comunidades }\end{array}$ \\
\hline & $\begin{array}{l}\text { Capacitar a la pobladión para } \\
\text { controlar y prevenir incendios }\end{array}$ & & $\begin{array}{l}\text { Protección Civil y las } \\
\text { Comunidades. }\end{array}$ \\
\hline & Realizar briga das preventivas. & & Protección Civil. \\
\hline & $\begin{array}{l}\text { Conseguir equipo para } \\
\text { controlar y prevenir incendios. }\end{array}$ & & $\begin{array}{l}\text { Protección Civil y las } \\
\text { Comunidades. }\end{array}$ \\
\hline \multirow{2}{*}{$\begin{array}{l}\text { Promover la cr eación } \\
\text { de normas muni cipales } \\
\text { destinadas a la } \\
\text { conservación, } \\
\text { restauración y al } \\
\text { manejo de los } \\
\text { bosques. }\end{array}$} & $\begin{array}{l}\text { Construir acu erdos para la } \\
\text { conservación, restauración y } \\
\text { uso de las zonas forestal es. }\end{array}$ & \multirow[t]{2}{*}{$\begin{array}{l}\text { Regidor de } \\
\text { Ecología y } \\
\text { Hacienda }\end{array}$} & $\begin{array}{l}\text { Regidor de Ecologí a y } \\
\text { Hacienda, Ejército, Comisariado } \\
\text { de Bienes Com unales, } \\
\text { Comisariado Ej idal y las } \\
\text { Comunidades. }\end{array}$ \\
\hline & $\begin{array}{l}\text { Acordar comprom isos y } \\
\text { sanciones para los delitos de } \\
\text { tala clandes tina. }\end{array}$ & & $\begin{array}{l}\text { Regidor de Ecologí a y } \\
\text { Hacienda, Ejérci to, Comisariado } \\
\text { de Bienes Com unales, } \\
\text { Comisariado Ej idal y las } \\
\text { Comunidades. }\end{array}$ \\
\hline
\end{tabular}


desde una perspectiva de planeación hecha por expertos (no construida por medio del consenso entre actores sociales, propia de la planeación colaborativa).

\section{Fase de foco}

Los resultados de esta fase demuestran que se abordó en forma satisfactoria. Asimismo, los resultados de las fases posteriores apoyan este argumento, ya que las potencialidades para contender con la conservación de los bosques en el municipio fueron evaluadas.

\section{Fase de análisis}

Los autores opinan que la metodología de Matus (1989), aplicada en esta fase, es eficiente para hacer una síntesis de las percepciones de los actores sociales, así como para analizar las potencialidades y la capacidad social para contender con problemáticas complejas como la de un plan de conservación forestal municipal. La aplicación de este enfoque metodológico permitió, de una manera fácil y sistemática, la identificación y descripción de los principales problemas relacionados con la conservación forestal. Asimismo, establecer con base en los resultados mostrados en tabla 2, lo que se consideró como la problemática para el cuarto pilar de MFS en el municipio "la falta de cultura acerca de la valoración de los recursos forestales en la población". Lo anterior coincide con los enfoques de Centro Europa para construir los indicadores de sustentabilidad. Estos indicadores cuando son enfocados a evaluar la potencialidad hacia la sustentabilidad, desde la perspectiva de la cultura, consideran fundamentalmente dos aspectos, la cultura de planeación local y la presencia de estrategias para el futuro. En la primera destacan los aspectos de carácter normativo, como normas y leyes de diferente ámbito (local, regional, federal) encaminadas a promover acciones para la protección del capital natural. En la segunda, el énfasis es la preocupación y compromiso de la comunidad en este mismo sentido (BLFUW, ca. 2000, Müller et al, 2000). A su vez, los autores consideran que como consecuencia de la problemática señalada anteriormente, se explican una serie de problemas asociados como la falta de un presupuesto suficiente para atender los aspectos de cuidado, protección y restauración del bosque y que las autoridades municipales no hayan considerado la creación y aplicación de un Programa de Conservación Forestal.

\section{Fase de diagnóstico}

Esta fase también se desarrolló eficazmente gracias a la metodología seleccionada. El árbol problemático descrito en la Figura 1, sintetiza la sinergia de causalidad entre los problemas identificados. A su vez, permitió la identificación de los nodos problemáticos: 1) La falta de presupuesto en el municipio destinado a la reforestación y conservación y 2) La falta de comunicación, concertación y coordinación entre las autoridades para el manejo de los recursos forestales.

Como se puede observar, la Figura 1 resume en forma gráfica el diagnóstico de las percepciones de la gente hacia el MFS. Asimismo, da lugar a la identificación rápida de aquellos problemas que deben ser prioritarios (problemas de raíz) que deben ser atendidos de inmediato si se pretende lograr un MFS, los cuales, a su vez, dan origen al proceso de intervención. Es decir, a la propuesta de una serie de acciones que se consideran estratégicas para poder contender con la solución de estos problemas.

\section{Fase de prognosis}

Nuevamente la metodología empleada fue adecuada, ya que permitió generar alternativas de solución para cada uno de los nodos detectados en el árbol problemático. La respuesta de estos autores a los nodos problemáticos se dio primeramente a través del establecimiento de dos ejes estratégicos: 1) Mejorar el presupuesto del municipio destinado a la conservación y reforestación y 2) Promover la coordinación, comunicación y concertación entre las diferentas autoridades para el manejo de los recursos forestales.

El primer eje se consideró bajo la 
premisa de que si se mejora el presupuesto se va a generar un mayor número de empleos. Lo anterior proporcionará los incentivos económicos necesarios para solucionar el problema de la escasa participación de la población para acciones destinadas a la conservación y reforestación del bosque y la poca valoración que se tiene del mismo.

Con respecto al segundo eje, éste se consideró porque al promoverse la coordinación, comunicación y concertación entre las autoridades municipales, es factible la resolución de los problemas relacionados con el reconocimiento oficial y operativo de los límites municipales y del manejo de los bosques en dicho municipio.

A partir de estos ejes se construyó un Plan de Acciones Prioritarias en donde se busca darle una forma operativa a las soluciones propuestas. Es por esto, que se decidió por una forma táctica que involucrara a los actores sociales identificados en la Tabla 1 , ya que tomarlos en cuenta a lo largo de todo el proceso de planeación se considera como uno de los factores clave para el logro del MFS en el municipio.

Análisis de amenazas y oportunidades

En términos generales, el análisis de amenazas y oportunidades deja ver un entorno poco optimista para la promoción local del MFS. Los resultados dejan ver por un lado una muy incipiente cultura sobre el valor de los bosques y una nula disposición a colaborar en su protección y conservación. Esto queda resaltado al ver que sólo dos actores sociales manifestaron su interés en el mismo: los Comisariados de Bienes Comunales y los regidores municipales. Los primeros se interesan en hacer y llevar a la práctica acciones encaminadas a construir un Programa de Aprovechamiento y Conservación Forestal. Estos actores sociales manifiestan particular preocupación por los factores locales que disminuyen la cubierta forestal de sus comunidades y por extensión del municipio. Por otra parte, los actores sociales ligados al gobierno municipal manifiestan interés en promover el uso diversificado del bosque, fundamentalmente orientado hacia el uso ecoturístico, aún a pesar de que se vea que esta promoción sólo beneficia a una comunidad del municipio.

Sin embargo, a pesar de esta disposición a colaborar, los resultados de la evaluación de amenazas y oportunidades dejan ver un panorama poco optimista para el logro del MFS. La apatía manifestada por la mayoría de los actores sociales y la falta de recursos humanos y financieros en el municipio llevan a pensar en lo difícil que sería echar a andar propuestas de sustentabilidad forestal. En opinión de los autores, los resultados comprueban que la disposición a colaborar es el factor clave para garantizar el éxito de este tipo de propuestas. Se piensa que aún consiguiendo los recursos para un plan de conservación forestal, si este no involucra el desarrollo de una cultura de conservación de los bosques y compromete a los habitantes de Tetela del Volcán, difícilmente tendrá una continuidad de largo plazo. Seguramente el proyecto moriría en cuanto los recursos financieros se terminaran.

\section{CONCLUSIONES}

La metodología propuesta permitió hacer una evaluación de las potencialidades para fomentar el MFS en el ámbito municipal. En este mismo sentido, también fueron clarificadas las percepciones que los diferentes actores sociales, involucrados en el manejo de los bosques, tienen sobre las necesidades de conservar los recursos forestales y las actividades que implicaría construir propuestas al respecto. La metodología propuesta por Matus (1989) dio lugar a una estructura epistemológica apropiada para representar y analizar, en una forma sistemática, los problemas en torno al MFS derivados de las encuestas. Gracias a su aplicación, los autores pudieron corroborar que las percepciones sociales sobre la conservación de los bosques son la principal restricción para fomentar localmente el MFS. De allí que, los autores hayan considerado que la problemática local para el logro del MFS sea "la falta de cultura acerca de la 
valoración de los recursos forestales en la población".

Con base en esta problemática, también se logró identificar lo que se denominaron como los nodos problemáticos. Es decir, aquellos problemas que por su importancia deberían ser atendidos en forma prioritaria para fomentar el MFS. Los autores consideraron a la falta de presupuesto, destinado a la reforestación (restauración) y conservación por parte del municipio, y a la falta de comunicación, coordinación y concertación entre diferentes autoridades para el manejo de recursos forestales, como los problemas a atender en forma inmediata si se quiere poner en marcha un manejo forestal encaminado hacia la sustentabilidad.

La forma de contender con estos dos nodos problemáticos se dió a través de una propuesta de planeación estratégica, la cual generó dos programas de acciones prioritarias. Estos dos programas se orientaron a: 1) Mejorar el presupuesto del municipio destinado a la conservación y reforestación y; 2) Promover la coordinación, comunicación y concertación entre las diferentas autoridades para el manejo de los recursos forestales. Si bien este trabajo sólo se queda en estas propuestas, los autores defienden la idea de que se ha identificado la información básica para desencadenar un futuro proceso colaborativo que dé lugar a un plan de manejo forestal orientado a la sustentabilidad.

\section{REFERENCIAS}

BLFUW, ca. 2000. Criteria catalogue for ecologically particularly sensitive areas. Federal Ministry of Agriculture, Forestry, Environment and Water Management. Viena, Austria.

Botequilha, A. y J. Ahern. 2002. Applying landscape ecological concepts and metrics in sustainable landscape planning. Landscape and Urban Planning 59:65-93.

Chávez-Cortes, J.M. 2004. Lansdcape planning to achieve sustainability: The Iztaccihuatl-Popocatepetl region, Mexico, as a case study. Tesis doctoral, The University of Liverpool, Liverpool, Reino Unido.

Christensen, N.L., A.M. Bartuska, J.M. Brown, S. Carpenter, C. D'Antonio, R. Francis, J.F. Franklin, J.A. MacMahon, R.F. Noss, D.J. Parsons, C.H. Peterson, M.G. Turner y R.G. Woodmansee. 1996. The report of the Ecological Society of America Committee on the scientific basis of ecosystem management. Ecological Applications 6(3): 665-691.

Constanza, R., R. d'Argue, R. de Groot, S. Farber, M. Grasso, B. Hannon, K. Limburg, S. Naeem, R.V. O'Neill, R.G. Raskin, P. Sutton y M. van den Belt. 1997. The value of world's ecosystem services and natural capital. Nature 387: 253-260.

de Groot, R.S., M.A. Wilson y R.M.J. Boumans. 2002. A typology of the classification, description and valuation of ecosystem functions, goods and services. Ecological Economics 41(3): 393-408.

Hartshorn, G.S. 1995. Ecological basis for sustainable development in tropical forests. Annual Review of Ecology and Systematics 26: 155-175.

Healey, P. 1997. Collaborative planning: shaping places in fragmented societies. UBC Press. Vancouver, Canada.

Higman, S., S. Bass, N. Judd, J. Mayers y R. Nussbaum, Eds. 1999. The sustainable forestry handbook. Earthscan Publications. Londres, Reino Unido.

Hill, T. y R. Westbrook. 1997. SWOT analysis: It's time for a product recall. Long Range Planning 30(1):4652.

Kajanus, M., K. Kangas y M. Kurttila. 2004. The use of value focused thinking and the A'WOT hybrid method in tourism management. Tourism Management 25: 
499506.

Kohn K. y J.F. Franklin. 1997. Creating a forestry for the 21st Century: the science of ecosystem management. Island Press. 475 p.

Lämas, T. y L.E. Ola. 2003. Analysis and planning systems for multiresource, sustainable forestry: The Eureka Research Programme at SLU. Canadian Journal of Forest Research 33:500-508.

Müller, F., R. Hoffmann-Kroll y H. Wiggering. 2000. Indicating ecosystem integritytheoretical concepts and environmental requirements. Ecological Modelling 130:13-23.

Matus, C. 1989. Política, planeación y gobierno. Altadir, Caracas, Venezuela.

Mendoza, G.A. y R. Prabhu. 2000. Multiple criteria decision-making approaches to assessing forest sustainability using criteria and indicators: a case study. Forest Ecology and Management 131:107-126.

Naiman, R., P.A. Bisson, R.G. Lee y M. Turner. 1997. Approaches to management at the watershed scale. In: K. Kohn y J.F.
Franklin, eds. Creating a forestry for the 21st Century: The Science of Ecosystem Management. Island Press, Washington, EUA. p. 239-254.

Pesonen, M., M. Kurttila, J. Kangas, M. Kajanus y P. Heinonen. 2001. Assessing the priorities using A'WOT among resource management strategies at the Finnish Forest and Park Service. Forest Science 47: 534541.

Ruelas Monjardín, L.C. 2004. A collaborative approach to water allocation in a coastal zone of Mexico. Tesis doctoral, The University of Liverpool, Liverpool, Reino Unido.

Selin, S. y D. Chavez. 1995. Developing a collaborative model for environmental planning and management. Environmental Management 19(2):189195.

Yaffee, S.L., A.F. Phillips, I.C. Frentz, P.W. Hardy, S.M. Maleki y B.E. Thorpe. 1996. Ecosystem management in the United States: an assessment of current experience. Island Press, Washington, EUA. 